\title{
Propositions pour une « approche combinée » des processus d'intégration économique régionale
}

L'Asie orientale comme test

Catherine Figuière et Laëtitia Guilhot

\section{OpenEdition}

Journals

Édition électronique

URL : http://journals.openedition.org/ei/5597

DOI : 10.4000/ei.5597

ISSN : 2553-1891

Éditeur

Association Économie et Institutions

Référence électronique

Catherine Figuière et Laëtitia Guilhot, « Propositions pour une « approche combinée » des processus d'intégration économique régionale ", Économie et institutions [En ligne], 20-21 | 2014, mis en ligne le 01 juin 2014, consulté le 30 avril 2019. URL : http://journals.openedition.org/ei/5597 ; DOI : 10.4000/ ei.5597

Ce document a été généré automatiquement le 30 avril 2019

Revue Économie et institutions 


\title{
Propositions pour une « approche combinée » des processus d'intégration économique régionale
}

L'Asie orientale comme test

\author{
Catherine Figuière et Laëtitia Guilhot
}

\section{Introduction}

1 Ce papier ${ }^{1}$ a pour objectif de montrer que l'analyse des processus régionaux, à la fois pris isolément -mais également à fin de comparaison-, ne peut faire l'économie d'une double démarche ou démarche combinée. Ainsi, les concepts produits par certains courants de l'Économie Politique Internationale (EPI), -comme ceux de « régime » ou de « bien public régional »-fournissent un cadre de réflexion pertinent pour rendre compte de la grande diversité des processus régionaux. Mais, si l'EPI, entendue ici comme une démarche globale, permet une interprétation de la spécificité de chaque processus, elle présente néanmoins une limite intrinsèque forte en ne proposant pas d'outils quantitatifs pour mesurer et qualifier, notamment, l'intensification des flux commerciaux dans une zone géographique donnée. Les chercheurs n'ont alors d'autre choix que de mobiliser les outils quantitatifs de l'économie internationale (EI), et d'interpréter les résultats dans le cadre préalablement établi par l'EPI. Cette "combinaison», forme maîtrisée "d'éclectisme méthodologique» (Gilpin, 2001) ne rencontre pas de contradiction épistémologique majeure, dans la mesure où les outils mis à contribution peuvent être considérés comme a-théoriques (Feenstra et al., 2001).

2 Il convient de préciser que si le cas régional est-asiatique ${ }^{2}$ est à l'origine de ce programme de recherche, il ne constituera ici que le moyen d'expertiser cette combinaison. Alors que la régionalisation dans cette zone s'intensifie avant la crise de 1997, à l'initiative des acteurs privés, ce sont les fortes perturbations des taux de change, à partir du mois de juillet 1997, qui vont faire émerger une demande de la part de ces acteurs en matière de stabilité monétaire et financière. Ce «bien public régional » ne pourra être "produit » 
que par la coopération interétatique entre les pays concernés par cette intensification des flux commerciaux. On assiste donc dans cette zone depuis les années 1990 à la complexification/intensification, tant des relations économiques entre acteurs privés, que des relations politiques entre Etats. Mais rien de clairement lisible -aucun accord de libre-échange ne vient par exemple regrouper l'ensemble des économies de la zonen'émerge (Guilhot, 2010), au moins jusqu'au début des années 2000, suite à la crise dite asiatique de 1997. Les périmètres économiques et politiques ne correspondent pas forcément. La compréhension de cette réalité régionale a donc suscité une réflexion sur la mise en place d'un appareillage méthodologique complexe, permettant de saisir dans une même analyse, les dimensions économiques et politiques. Ce programme correspond à un processus itératif entre la construction des faits stylisés du cas asiatique et la mise au point d'une méthodologie ayant pour objectif «d'attraper» toutes les formes de processus régionaux.

3 Afin d'organiser les résultats obtenus, la première section précisera la convention sémantique retenue pour qualifier les différents aspects d'un processus d'intégration économique régionale (IER). La deuxième section s'attachera d'une part, à différencier les approches en EPI, et, d'autre part, à préciser la valeur explicative des concepts fournis par le courant néo-libéral de l'EPI (désormais EPINL) adopté ici pour appréhender la diversité des processus régionaux. Dans la troisième section, les outils quantitatifs de l'EI seront appréhendés comme un complément compatible et nécessaire de l'analyse en EPINL, pour mesurer la régionalisation.

\section{Intégration économique régionale : quelle convention sémantique?}

4 Il convient dans un premier temps de se doter d'une convention sémantique permettant de rendre compte des dimensions politiques et économiques de l'intégration, afin d'en bien saisir la combinaison. Ces propositions seront ensuite appliquées au processus régional est-asiatique. Ce point ne prétend à aucun apport conceptuel et n'a donc aucune portée normative. Sa seule ambition est de permettre de progresser dans la comparaison entre les différents processus régionaux en proposant de fixer l'utilisation des termes dédiés.

\subsection{De la régionalisation à l'IER en passant par le régionalisme... quels termes pour quelles réalités régionales?}

5 Le foisonnement des termes dans la littérature tant francophone qu'anglophone oblige à un détour sémantique en préalable à toute analyse du fait régional (Hugon, 2001). La nécessité s'impose de retenir une convention qui permette de rendre compte des deux aspects de l'intégration régionale : la concentration des flux économiques dans une zone, d'une part, l'institutionnalisation des relations interétatiques, d'autre part. En effet, les 
deux processus ne progressent pas nécessairement sur les mêmes périmètres ni selon les mêmes calendriers.

La convention arrêtée ici retient trois termes: régionalisation, régionalisme et intégration économique régionale (IER), dont les contenus vont être définis de la façon suivante (Figuière et Guilhot, 2007) :

- La régionalisation qualifie une concentration des flux économiques entre nations géographiquement proches, plus que proportionnelle à la croissance des flux avec le reste du monde.

- Le régionalisme qualifie exclusivement l'institutionnalisation des relations interétatiques entre nations géographiquement proches.

- Par suite l'intégration économique régionale correspond strictement à une combinaison de régionalisation et de régionalisme.

7 Le processus de régionalisation dessine un périmètre économique, alors que le processus de régionalisme dessine un périmètre politique. Le périmètre de l'IER dans une zone géographique donnée sera déterminé par l'intersection entre les deux précédents.

8 A ces définitions consubstantielles des fondamentaux du fait régional, viennent s'ajouter des variations sur le thème de l'IER. En effet, celle-ci peut se faire « en surface », « en profondeur » ou aller jusqu'à pouvoir être qualifiée de « supranationale » (Guilhot, 2008).

- L'intégration en surface (shallow integration) vise à instaurer des règles communes portant sur les relations entre les nations. L'ASEAN avec son accord de libre-échange -régulation des flux commerciaux aux frontières- en est l'illustration.

- L'intégration en profondeur (deep integration) a pour but d'harmoniser les pratiques au sein de chaque nation impliquée. L'ALENA ${ }^{3}$ ne se limite pas à une libéralisation des échanges commerciaux ; le secteur des services, des marchés publics et la réglementation sur les IDE (chapitre 11 de l'accord) sont aussi concernés. Une harmonisation des normes s'effectue notamment sur la base de la nation la plus favorisée et du traitement national.

- L'intégration supranationale suppose un transfert de souveraineté de l'une au moins des prérogatives préalablement réservées aux États nationaux. La création de l'euro, monnaie unique gérée par la Banque Centrale Européenne, constitue la seule illustration à ce jour de cette dernière forme d'IER.

Le graphe suivant synthétise les différentes combinaisons régionales envisageables, tout en illustrant la possibilité de s'émanciper d'un déterminisme historique à la Balassa, dont le « modèle » s'inspire largement du cheminement européen. Or, l'histoire montre qu'un processus régional peut très bien ne pas entamer son institutionnalisation par un accord de libre-échange et, de fait, viser directement l'intégration en profondeur, sans nécessairement passer par la phase d'intégration en surface. 


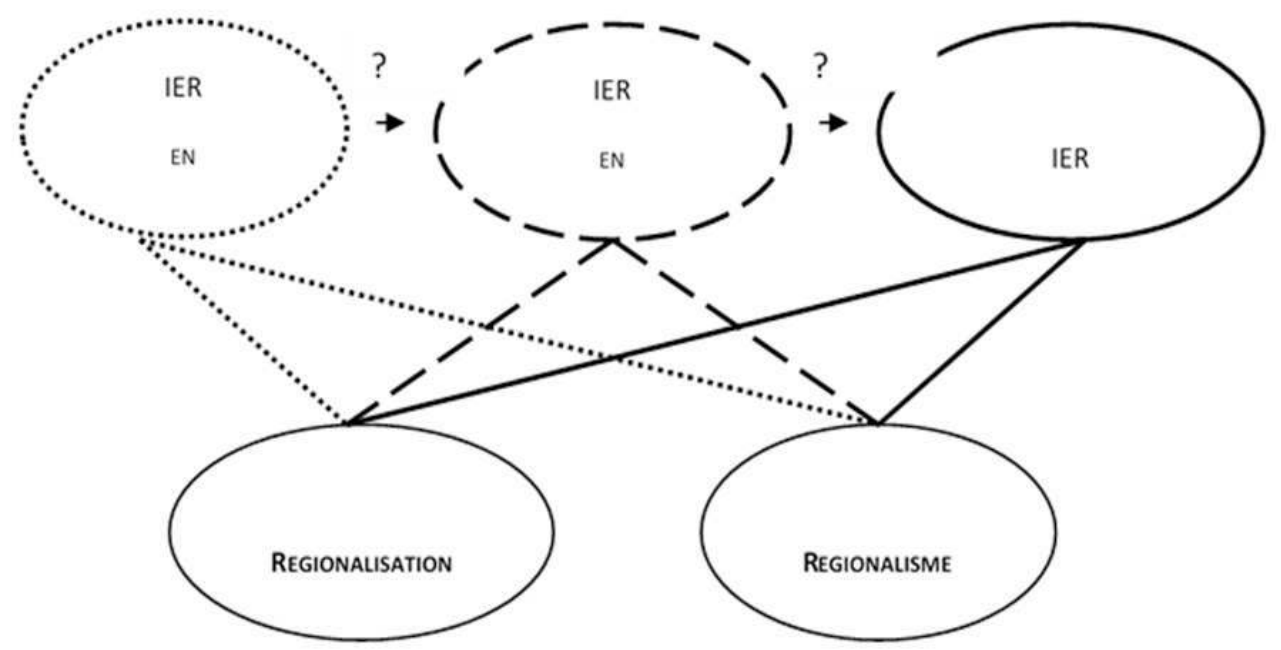

Dans la mesure où le cas est-asiatique a été retenu pour tester la proposition portée par ce texte, il convient de le positionner en fonction de cette convention sémantique.

\subsection{Régionalisation et régionalisme en Asie Orientale : quelles combinaisons?}

L'Asie Orientale rassemble les économies les plus dynamiques du monde depuis plusieurs décennies, elle constitue donc un objet d'étude intéressant du fait régional.

Le seul périmètre politique existant en Asie Orientale, avant la crise de 1997, est celui que dessine l'Association des Nations d'Asie du Sud Est (ASEAN) créée en 1967. Cette association se met en place sur une logique de «ni-ni»: ni les américains, ni le communisme (Boisseau du Rocher, 1998). Le respect des souverainetés nationales constitue le pivot de ce « régionalisme politique léger». Les motivations initiales sont en effet très clairement orientées davantage vers la politique -le maintien de la paix dans la zone- que vers l'économie, bien que la promotion de la croissance des pays membres figure dans le texte fondateur. Cette forme de coopération se définit davantage comme un état d'esprit -the Asean way- que comme la mise en place d'un véritable régionalisme. Il faudra attendre 1992, et l'instauration d'une zone de libre-échange entre les membres (AFTA, Asean Free Trade Area), pour pouvoir parler «d'harmonisation des règles en surface ». Une charte, ratifiée en 2008, vient compléter ce dispositif.

Entre 1967 et le début des années 2000 (avec l'Initiative Chiang Mai en 2000), l'ASEAN dessine donc le seul périmètre politique de la zone, et constitue également un espace de régionalisation puisque les échanges à l'intérieur de la zone augmentent plus rapidement qu'avec le reste du monde (cf. point 3). Néanmoins, à partir des années 1990, un autre regroupement économique s'affirme avec l'augmentation des relations entre les membres de l'ASEAN et ses voisins d'Asie du Nord-Est: le Japon d'abord, puis la Corée du Sud, et enfin la Chine, qui s'ouvre petit à petit pour finir par adhérer à l'OMC en 2001.

Dès les années 1990, le dynamisme des échanges au sein de ce nouvel ensemble supplante celui des échanges intra-ASEAN. Un nouvel espace de régionalisation s'impose donc. Néanmoins, l'ASEAN reste le seul périmètre politique puisqu'aucun accord de libre- 
échange ne vient accompagner la régionalisation de l'ASEAN + 3. Il faut attendre la crise de 1997 pour que d'un dialogue, d'abord informel, émerge une véritable institutionnalisation des relations interétatiques entre les 13 membres de l'ASEAN + 3. Ce périmètre politique se construit non pas sur la volonté de mettre en place des règles communes concernant les échanges en eux-mêmes, mais sur ce qui les rend possibles : la monnaie et la finance. La zone s'émancipe donc du calendrier « traditionnel » « Trade first, money later " (Plummer et Wignaraja, 2006) suivi par l'Union Européenne. La méthode proposée dans ce texte devrait, également, permettre de répondre à la question «Pourquoi cette « spécificité » asiatique?»

\section{L'EPI comme cadre théorique}

15 L'EPI est la seule approche qui permet aux économistes d'embrasser dans une même analyse des données strictement économiques et des données politiques. Selon la définition de Kébabdjian (1998: 103) l'EPI, constitue « une tentative interdisciplinaire cherchant à analyser la sphère des relations économiques internationales, centrées sur les phénomènes de richesse, en prenant en compte les articulations avec la sphère du politique, centrée sur les phénomènes de pouvoir. (...) Deux courants traversent aujourd'hui la théorie "orthodoxe»: le courant néo-libéral, qui met l'accent sur les relations d'intérêt (richesse); le courant néo-réaliste qui met l'accent sur les relations de pouvoir (puissance).» Cette démarche originellement initiée par les politistes anglosaxons après la Seconde Guerre Mondiale (Chavagneux, 2010), est aujourd'hui structurée par une multitude de courants qui peuvent schématiquement être classés en deux grandes tendances : les analyses «standards " (ou orthodoxes si on se réfère aux travaux de Kébabdjian, 2006), et les analyses "critiques». La démarche retenue ici pour caractériser un processus d'intégration régionale prend son fondement dans l'un des courants standards : l'institutionnalisme néolibéral (Keohane, 1984, et Krasner, 1983). Il convient de rappeler ici que le «néolibéralisme en EPI n'a qu'une parenté lointaine avec ce qu'on appelle habituellement chez les économistes, néolibéralisme et "politiques néolibérales ». (...) Le néolibéralisme désigne toute autre chose en EPI. (Cette) nouvelle approche, apparue dans les années 1980, a développé une théorie institutionnaliste complète de l'économie politique internationale, à partir de l'idée des «échecs du marché » » (Kébabdjian, 1998 :38).

Cette approche s'impose ici pour deux raisons majeures: l'absence de conflit épistémologique avec l'économie internationale dont sont issues les méthodes quantitatives mobilisées ici, d'une part, la nature interétatique des relations de coopération sous examen dans les zones concernées, d'autre part. Elles visent en effet à satisfaire un besoin collectif non atteignable par un Etat isolé et recherché par une coalition d'Etats - approche néolibérale- et non à améliorer la puissance d'un ensemble d'Etats dont l'action serait coordonnée par le pays hégémonique - approche néoréaliste. Par la suite, le recours au cas asiatique permettra de mieux préciser la démarche. Dans la mesure où la coopération interétatique multilatérale, en d'autres termes, la construction d'une architecture institutionnelle, en Asie Orientale progresse davantage dans les champs monétaire et financier, la démarche en EPINL ne sera déclinée que dans ce domaine. Néanmoins, le raisonnement est parfaitement transposable au régionalisme commercial. 

régional portés par l'EPINL, l'un des courants standards de l'EPI, qui vont donc être mobilisés. Ils autorisent en effet la focalisation sur les relations interétatiques et donc sur l'institutionnalisation des relations entre les pays d'une même région. Ils permettent également de rendre compte de la grande diversité des cheminements vers l'intégration régionale. En effet, autorisant notamment un recours à l'histoire, contrairement à des analyses plus statiques, cette démarche va permettre d'« expliquer » la "spécificité » de chaque processus dans un contexte international daté. Dans ce cadre, l'Europe s'est ainsi construite dans une phase où le régime commercial était balbutiant, mais les institutions de Bretton Woods, toutes puissantes: elle a donc commencé par se doter d'un régime commercial, un environnement commercial stabilisé et pacifié constituant le bien public international alors recherché. Ce bien public n'étant pas disponible à l'échelle internationale, c'est sur une base régionale que l'Europe le produit («trade first»), satisfaisant sur cette base géographique la demande d'un bien public qui devient, de fait, régional. La création de l'euro correspondra, par la suite, à la mise en place d'un régime monétaire régional, pour faire face, d'une part, à l'instabilité du régime monétaire international dans le domaine des taux de change ("money after»); d'autre part, au recours au dumping monétaire de certains partenaires européens («free riding»). Le calendrier asiatique diffère du calendrier européen, parce que le contexte international diffère, c'est l'actualité (la crise de 1997, puis celle de 2008) qui impose le déroulement de la construction asiatique. Le délitement du système monétaire et financier contraint les Etats à coopérer dans le domaine monétaire et financier ( money first »), alors que la pérennité du régime commercial -sous l'égide de l'OMC- leur permet de se passer d'un accord commercial régional multilatéral («trade after »). La multiplication des accords de libre-échange bilatéraux ou englobant seulement certaines économies de la région comme par exemple, l'AFTA (Asean Free Trade Area) ou l'accord Chine-ASEAN, fait penser au «spaghetti bowl» décrit par Bhagwati (2002) ${ }^{4}$, mais ne constitue pas à ce jour de cadre commercial multilatéral dans cette zone. deux types d'éléments de natures très différentes -des flux économiques d'une part, un construit institutionnel de l'autre-; requiert une méthodologie complexe combinant explicitement des emprunts faits à deux corpus. L'EPINL va fournir le cadre conceptuel de la réflexion, les méthodes quantitatives de l'EI viendront compléter ce corpus de référence -qui ne dispose pas d'outils de mesure du fait international/régional- en proposant notamment une mesure des flux économiques constitutifs de la régionalisation.

\subsection{La stabilité monétaire et financière comme bien public régional (BPR) : quelle pertinence?}

La présente tentative de mobilisation d'une EPINL pour analyser à la fois la diversité des processus régionaux, mais aussi la réalité «politique» de chacun, a été initiée par les répercussions de la crise dite «asiatique » ou « de 1997 ». Les acteurs privés de la zone ayant au préalable amorcé un processus de régionalisation « naturelle » (Krugman, 1991) c'est-à-dire initié par des acteurs privés- (cf. point 3), la crise des taux de change fait émerger un besoin de stabilisation des conditions des échanges, et donc une demande de stabilité monétaire et financière. Elle révèle dans le même temps, l'incapacité du système 
monétaire et financier international à satisfaire ce besoin. L'objectif que vont alors chercher à atteindre les Etats de la zone est celui de la mise en place de la production de cette stabilité sur une base régionale. Dès lors, le concept de «bien public international » (Kindleberger, 1986) peut être utilement mobilisé. Dans la mesure où l'organisation de la production d'un tel bien nécessite une coopération internationale, l'analyse montrera la pertinence de la mobilisation du concept de «régime international » pour progresser dans la compréhension de cette production.

En préalable à l'application du concept de BPR au cas asiatique, trois précisions doivent être apportées :

1. La stabilisation économique, financière ou monétaire constitue explicitement un bien public international pour de nombreux auteurs, dont Stiglitz (1999), prix Nobel d'économie depuis.

2. Si le concept de bien public a été initialement conçu dans le cadre des économies nationale, son élargissement "géographique » à l'échelon international par l'EI, est allé de pair avec son " élargissement théorique » : « la notion de bien public, même si elle trouve ses sources dans l'analyse standard, devient avec son extension aux biens publics mondiaux un objet de débats et d'approches différentes, tant dans la définition du contenu du bien public que dans la mise en œuvre des programmes concernés » (Boidin et Zuindeau, 2006:29). Gabbas et Hugon (2001:27) mentionnent la richesse interprétative de la mobilisation de ce concept dans un cadre d'économie politique internationale.

3. Le concept de «bien public » peut être transposé à l'échelle régionale (Aubin, 2005). Des institutions n'hésitent pas à adopter cette démarche, ainsi, dans son programme de «stratégie d'intégration régionale pour l'Afrique australe 2011-2015» (en ligne sur le site), la Banque Africaine de Développement fait explicitement référence à la nécessité de produire des «Biens publics régionaux». Des chercheurs proposent également une lecture de la production des biens publics sur une base régionale. Benaroya $(2002: 431-432)$ mentionne notamment «la gestion des biens publics à l'échelon régional», tout en soulignant que « rares sont les travaux qui se sont intéressés aux organisations régionales (... ) en cherchant à cerner leur capacité à fournir la gamme des principaux biens internationaux ». Si le concept de bien public peut être transposé à l'échelon régional, alors le concept « lié » de régime (tout comme celui de leadership) le peut également.

21 La construction institutionnelle est-asiatique vise la stabilité monétaire comme bien public régional. Cette stabilité fait référence à la recherche de stabilité du prix des actifs, en particulier la stabilité des taux de change (tout comme la mise en place de l'euro peut être considérée comme un bien public régional, visant à éradiquer les impacts des fluctuations erratiques de change entre des partenaires économiques très interdépendants, ainsi que la manipulation de ces taux en vue d'améliorer la compétitivité prix des exportations). La stabilité financière fait, quant à elle, référence à la stabilité des institutions financières (établissements bancaires et non bancaires) et des marchés financiers (Crockett, 1997). Elle constitue un corollaire indispensable de la stabilité monétaire.

La crise de 1997, en tant que telle, mais aussi l'inaction de l'ASEAN et de l'APEC, ou encore les politiques inappropriées du FMI, ont profondément marqué la population et les gouvernements des pays est-asiatiques. Pour reprendre les propos de Beeson (2003: 266), " the crisis revealed both the continuing vulnerability of the region as a whole to external leverage and the lack of a regional capacity to manage such events ». La coopération durable, informelle puis formelle, qui se met en place dès le mois de décembre 1997 entre les gouvernements de la région, peut dès lors être lue comme la manifestation d'une volonté explicite de produire à l'échelle régionale de la stabilité monétaire et financière. Si le périmètre 
pertinent de production constitue ex ante l'un des grands problèmes inhérents à la production des biens publics internationaux (dès lors qu'ils ne sont pas globaux) (Kébabdjian, 1994), le contour de la zone asiatique va se dessiner aisément pour au moins trois raisons :

1. La crise qui vient de toucher gravement la plupart des économies de la zone (Nicolas, 2006) par un effet de contagion.

2. L'intensification préalable des flux intra-zone qui augmente l'interdépendance entre les pays de la zone implique y compris les économies peu touchées par la crise (dont la Chine, Bresling, 2008).

3. La panne du multilatéralisme qui fait que des pays comme le Japon voit dans le régionalisme la solution d'avenir pour la coopération interétatique (Boulanger, 2006).

Cherchant à organiser la production de la stabilité monétaire et financière sur une base régionale, les pays d'Asie répondent à une injonction théorique assez répandue dans la littérature. Nombreux sont en effet les auteurs qui préconisent cette échelle de production (Cook et Sachs, 2002 ; Wyplosz, 2002 ; Ocampo, 2002 ; Kawai, 2004). Les effets de contagion entre les pays appartenant à une même zone étant importants, ils peuvent impulser une dynamique qui pousse les gouvernements régionaux à coopérer, afin de mettre en place une certaine harmonisation de leurs réglementations financières et des mécanismes de surveillance afin de tenter d'éviter de nouvelles crises, ou, tout au moins, d'en limiter les impacts.

La diffusion de la crise "hypothécaire" américaine à l'ensemble du monde, et en particulier à l'Asie, suscite un regain d'intérêt pour la démarche régionale (Figuière et Guilhot, 2011). Preuve, s'il en est, de l'échec du système financier international, cette contagion n'a pas manqué de provoquer une accélération du processus de régionalisme asiatique avec la réapparition du projet de Fonds Monétaire Asiatique dès octobre 2008 (cf. point suivant).

Une fois établie la pertinence de la mobilisation du concept de bien public régional pour qualifier la stabilité monétaire et financière en Asie Orientale, la généralisation de cette proposition à d'autres zones est immédiate. Désormais, les modalités de la production de ce «bien » doivent être précisées.

\subsection{Le concept de régime comme mode de production d'un bien public régional}

Kébabdjian (1998:107) considère que la proposition suivante traduite de Krasner (1983) constitue désormais la définition canonique du « régime international » : « des ensembles explicites ou implicites de principes, de normes, de règles, et de procédures de prise de décision autour desquelles les anticipations des acteurs convergent dans un domaine donné des relations internationales ». Il propose de compléter cette définition par la considération de trois déterminations :

- "Les régimes internationaux définissent d'abord un ordre partiel», en d'autres termes un ordre s'appliquant à un objet donné des relations internationales: le commerce, les taux de change, le climat, etc. (given area ou issue area)

- Un régime est en deuxième lieu un "ordre taxinomique" en cloisonnant très nettement le traitement de chaque question : ainsi le « régime commercial » ne se préoccupe en aucun cas des taux de change qui relève du « régime monétaire ». 
- Un régime est en troisième lieu « un ordre institutionnel », doté d'une « loi commune », limitant la souveraineté des États participants, et améliorant les performances du système pour chaque État.

L'EPINL identifie généralement deux modes de production pour les biens publics internationaux (Guilhot, 2009) : l'existence d'un leader effectif ou la création d'un régime. Transposée, là encore, à l'échelle régionale, et à partir du constat de l'absence d'un leader dans la zone étudiée ${ }^{5}$, cette proposition amène à considérer les étapes de la coopération interétatique en Asie comme correspondant à la volonté d'instauration d'un régime régional. Avant de qualifier plus précisément ce régime, l'encadré suivant, en précisant les grandes étapes de la coopération interétatique instituée en réaction à la crise de 1997, met l'accent sur la réalité «thématique » de ce régime. La focalisation des deux grandes initiatives asiatiques, l'Initiative Chiang Mai (ICM) et l'Asian Bond Markets Initiative (ABMI) sur les questions monétaires et financières permet sans contestation possible de qualifier ce régime de «monétaire et financier». En effet, si, de prime abord, ces avancées semblent porter uniquement sur des aspects financiers, la question monétaire dans sa dimension internationale, reste bien l'objectif ultime.

Encadré 1 . Les mesures fondatrices de la formation d'un régime monétaire et financier régional en Asie Orientale

1997 Premier sommet informel entre les 13 pays à Kuala Lumpu

2000 2ème Réunion des Ministres des Finances de l'ASEAN+3 en Thaïlande. Instauration de l'Initiative Chiang Mai (ICM).

2003 Création de l'ABMI (Asian Bond Markets Initiative) afin de développer les marchés obligataires est-asiatiques et du Research Group (sur proposition japonaise) qui a pour but d'explorer les différents moyens pour renforcer la coopération financière et promouvoir la stabilité dans la région en s'appuyant sur les travaux académiques des différents pays membres.

2005 Mai $8^{\mathrm{e}}$ réunion des Ministres des Finances à Istanbul. Accord pour améliorer l'effectivité de l'ICM. L'ASEAN+3 Economic Policy Review and Dialogue Process est intégré à l'ICM. Adoption d'un mécanisme de décision collective pour l'activation des swaps. Les liquidités de court terme, mises à disposition des pays sans l'appel du FMI passent de 10 à $20 \%$. Une multilatéralisation de l'ICM est envisagée.

$20069^{e}$ Réunion des Ministres des Finances de l'ASEAN+3, renforcement de l'ICM. Création de deux groupes de travail, Group of Experts et ETWG (Technical Working Group on Economic and Financial Monitoring) pour améliorer la surveillance macroéconomique de la région.

2007 Mai $10^{e}$ Réunion des Ministres des Finances de l'ASEAN+3 : la mise en commun des réserves est considérée comme la forme appropriée pour multilatéraliser l'ICM. Les députés sont chargés d'étudier les éléments clés pour que cette multilatéralisation puisse avoir lieu, c'est-à-dire, la surveillance, les mécanismes d'activation, les quotas d'emprunt.

2008 Mai Lors de la $11^{\mathrm{e}}$ Réunion des Ministres des Finances de l'ASEAN+3, les Ministres s'accordent sur un montant des réserves mises en commun (au moins 80 milliards de dollars), les conditions d'accessibilité des emprunts et le mécanisme d'activation. Les pourparlers sur la répartition des contributions se précisent également : ils annoncent une répartition « 20:80 » entre les pays de l'ASEAN et les trois autres économies (Chine, Japon et Corée du Sud). 
2009 Décembre Face à la diffusion de la crise financière, the Chiang Mai Initiative devient véritablement « multilatérale » (CMIM) et se voit désormais dotée de tous les attributs d'un Fonds Monétaire Asiatique (FMA) : multilatéralisme régional, système de surveillance et dotation initiale de 120 milliards de dollars.

2010 Mars L'accord venant entériner le projet de CMIM est signé par les 13 pays. L'AMRO (ASEAN+3 Macroeconomic Research) Office est créé. 2011 Avril L'AMRO est opérationnel. http://www.amro-asia.org/ 2012 Mai Approfondissement de l'ABMI avec l'instauration du « New Roadmap + » et du CMIM avec le renforcement du mécanisme de résolution des crises, qualifié de CMIM-SF (Stability Facility) (doublement des fonds : 240 milliards de dollars) et amélioration du mécanisme de prévention des crises, qualifié de CMIM-PL ( Precautionary Line)

À partir notamment de Sussangkarn (2010), Yuan et Murphy (2010), ADB (2009) et du site de l'ASEAN

La coopération interétatique qui s'instaure à partir de décembre 1997 (Guilhot, 2009) relève très clairement d'une volonté commune de résolution d'un problème d'action collective - et non d'une logique de normalisation. En effet, la nature de la crise de 1997 fait prendre conscience aux dirigeants politiques de la zone que leurs économies nationales ont à faire face à un problème commun : l'instabilité des taux de change et les mouvements erratiques de capitaux qui en découlent, auxquels ils ne peuvent faire face que collectivement. La coopération leur permettrait de produire de la stabilité (BPR) qui leur est inaccessible unilatéralement. Cette situation correspond a priori à la mise en place d'un régime de collaboration (Stein, 1983). Ici la coopération ne constitue pas une fin en soi, elle n'est que le moyen de résoudre le problème via la production du bien public régional. Il convient par ailleurs de souligner que ce régime, régional, a pour objectif explicite de compenser la faillite du régime international.

Le contenu des mesures et des lois mises en place dans ces économies, laisse à penser qu'un processus d'harmonisation des pratiques se met en en place. En effet, les pays de la région adoptent de nouvelles lois afin de se conformer aux objectifs de régulation de ce régime. Etant donnée l'hétérogénéité des niveaux de développement des pays impliqués et le pragmatisme caractérisant les économies est-asiatiques, cette harmonisation n'est pas encadrée formellement par une loi. Son but ainsi n'est pas de contraindre les pays estasiatiques à intégrer stricto sensu des pratiques mais de leur fournir, dans un premier temps, une assistance technique afin d'évaluer leur situation économique et politique, puis de proposer les mesures nécessaires à mettre en place selon les pays. Son caractère informel permet à chaque pays d'intégrer les réformes à son rythme mais ne laisse pas présager si l'ensemble des pays va continuer à mettre en place ces réformes.

Ce processus d'harmonisation se constate par l'instauration de nouvelles lois dans certains pays est-asiatiques en conformité avec l'objectif de développement des marchés obligataires réalisé dans le cadre de l'ABMI. Par exemple, en 2006 le Cambodge adopte de nouvelles lois afin de développer son marché obligataire, incluant une loi sur les obligations d'État et d'entités non étatiques. La Chine autorise, quant à elle, le développement de nouveaux produits comme les swaps de taux d'intérêt. Le gouvernement chinois souhaite continuer à améliorer son marché et réfléchit actuellement à l'introduction de nouveaux émetteurs, de nouveaux produits et à l'établissement d'un système de supervision. Les mesures prises par le gouvernement 
thaïlandais vont également dans ce sens. Elles permettent aux non-résidents un plus grand accès au marché obligataire national. Les non-résidents investissant dans les obligations d'État sont exemptés d'une taxe forfaitaire. Le gouvernement thaillandais autorise, par ailleurs, des entités étrangères installées dans un des pays des treize pays de l'ASEAN+3 à émettre des obligations en Baht (AFMM, 2006). Dans le cadre de l'ABMI, les programmes d'assistance technique à l'Indonésie, au Laos, à la Birmanie et au Vietnam viennent d'être achevés. Les Philippines continuent quant à elles de bénéficier de cette assistance afin de faire progresser les infrastructures de son marché obligataire (Site Amro).

\subsection{La crise de 2007 , accélérateur de la construction d'un régime régional.}

31 L'observation détaillée de l'activisme institutionnel suscité par la crise de 2007, permet de progresser dans la validation de l'hypothèse de la constitution d'un régime monétaire et financier asiatique. Ainsi, masqué derrière des appellations parfois (volontairement?) trompeuses, le processus d'institutionnalisation de la coopération monétaire en Asie a connu en 2010 une avancée très significative, en réponse à la diffusion de la crise des subprimes. En effet, qui irait voir derrière «la multilatéralisation de l'Initiative Chiang Mai », la mise en place d'un véritable Fonds Monétaire Asiatique (FMA) (Henning, 2009) ? De même, l'ABMI progresse dans l'approfondissement des marchés obligataires estasiatiques.

Le projet de FMA, qui regroupe les 13 pays de l'ASEAN+3 (Plummer, 2010 ; Winkler, 2010 ; Jetin, 2009), a été proposé une première fois par le Japon dès 1998, rencontrant alors une vive opposition de la part des États-Unis et de la Chine (Higgott, 1998 ; Hamanaka, 2009) ${ }^{6}$. Dix ans plus tard en pleine crise des subprimes, ce projet ressort des cartons comme suite logique à la construction institutionnelle en cours dans la région (ADB, 2010b). L'interdépendance commerciale régionale incite en effet la Chine à jouer désormais la carte de la stabilisation régionale. De plus, la Chine sait souffrir d'une image encore perfectible auprès de ses voisins et continuerait ainsi à redorer son blason, comme elle avait notamment commencé à le faire en ne dévaluant pas lors de la crise de 97-98, afin de ne pas aggraver les difficultés des autres pays asiatiques (avec qui elle entretenait alors des relations commerciales nettement moins intenses).

33 Après avoir été écarté pendant près de dix ans, ce projet revient sur le devant de la scène, en empruntant la voie de la multilatéralisation de l'ICM, initiée en 2000. Face à une situation économique mondiale de plus en plus préoccupante, en mai 2008, lors de la déclaration conjointe des Ministres des Finances, les pays s'engagent à accélérer les discussions afin d'arriver à un consensus sur les modalités de la multilatéralisation de l'ICM, basée jusque-là sur des accords bilatéraux. Les treize Ministres s'accordent sur le montant des réserves mises en commun (au moins 80 milliards de dollars, soit le montant alors couvert par le réseau de swaps), les conditions d'accessibilité des emprunts et le mécanisme d'activation. Les pourparlers sur la répartition des contributions progressent également: on s'oriente vers une répartition « 20/80 » pour les pays de l'ASEAN et les trois autres économies (Chine, Corée du Sud et Japon).

La déclaration des treize Ministres de l'ASEAN+3 lors du 12e sommet, le 3 mai 2009 à Bali, confirme les conditions de cette multilatéralisation et son instauration d'ici la fin de l'année. Les pays s'accordent ainsi sur le montant alloué (qui passe de 80 à 120 milliards 
de dollars), sur le mécanisme de surveillance et sur les contributions de chaque partenaire : (32\% pour la Chine et le Japon (38,4 Mds chacun), 16\% pour la Corée du Sud (19,2 Mds), 20\% pour les dix pays de l'ASEAN dont $95 \%$ sont fournis par les cinq membres fondateurs: Thaillande, Philippines, Indonésie, Malaisie, Singapour. Les treize pays auxquels se joint l'autorité monétaire de Hong Kong - tiennent leurs engagements. Le 28 décembre 2009, la multilatéralisation de l'ICM (CMIM en anglais) est signée après de longues négociations. Elle entre en vigueur le 24 mars 2010 (Siregar et al., 2013). Malgré cette multilatéralisation, des accords de swaps bilatéraux entre les treize économies demeurent. Ils sont désormais envisagés comme des compléments aux fonds alloués par la CMIM.

Parallèlement à la volonté de multilatéraliser les accords de swaps (originellement bilatéraux lors de la signature de l'ICM en 2000), les treize pays décident de renforcer le mécanisme de surveillance de l'ICM afin d'éviter le problème d'aléa moral inhérent à un accord strictement « régional» (ADB, 2009). Suite à la déclaration conjointe des Ministres des Finances, du 4 mai 2008, les dirigeants de l'ASEAN+3 mettent en place des mesures visant à renforcer l'ERPD (ASEAN+3 Economic Review and Policy Dialogue). Les trois mesures phares sont l'augmentation des fréquences des dialogues et le développement d'un format standardisé pour les rapports d'information, mais surtout, la création de l'AMRO (ASEAN+3 Macroeconomic Research Office) (ADB, 2010a).

L'AMRO (2010-2011) vient renforcer l'ERDP (qui date de 2000) principalement en matière de surveillance macroéconomique (Sussangkarn, 2010). Sa création marque ainsi un tournant important dans l'histoire de l'ASEAN+3, qui ne compte toujours pas de secrétariat permanent. L'AMRO, basé à Singapour, compte en effet une dizaine de permanents. La seizième Déclaration conjointe des Ministres des Finances et des Gouverneurs des Banques Centrales de l'ASEAN+3, en mai 2013, annonce la transformation de l'AMRO en « organisation internationale » (site de l'AMRO).

En mai 2012, lors de la réunion des gouverneurs des Banques Centrales et des Ministres des Finances des pays de l'ASEAN+3, la décision de renforcer la CMIM par deux initiatives, a été prise. La première a pour but de renforcer le mécanisme de résolution des crises, qualifié de CMIM-SF (Stability Facility), en doublant le fonds (240 milliards de dollars désormais), en limitant l'appel à l'intervention du FMI et en instaurant un contrôle des économies sur une base biannuelle. La seconde instaure un mécanisme de prévention des crises, qualifié de CMIM-PL (Precautionary Line) qui inclut cinq critères de qualification : 1/ la position externe et l'accès au marché, 2/ la politique fiscale, 3/la politique monétaire, 4 / la solidité du secteur financier et sa supervision, $5 /$ la date adéquate (site de l'ASEAN, déclaration conjointe).

Le renforcement progressif de l'ICM (offre de ressources et mécanismes de surveillance) présente des ressemblances étonnantes avec la création d'un "Fonds Monétaire Asiatique » (Chey, 2009 ; Henning, 2009), appellation du projet originel nippon de 1998. Si ce terme n'est pas (encore) employé officiellement par les treize gouvernements, le renforcement du mécanisme de surveillance et la multilatéralisation des accords de swaps permettent d'affirmer qu'un FMA a bien été créé en mars 2010. Les asiatiques se défendent néanmoins de vouloir concurrencer le FMI, tout en soulignant que cela permet aux pays de bénéficier de lignes de crédit jusqu'à un certain seuil ${ }^{7}$, sans tomber sous les critères de conditionnalité du FMI : «Under the CMIM, a country can draw upto 20\% of its quota for up to six months without being subject to IMFconditionality, while any amount over this limit would need to be tied to an IMF program» (ADB 2010a:76). Il n'est pas inutile de 
rappeler ici combien les asiatiques ont mal vécu les ingérences du FMI lors de la crise du 1997. La position des États-Unis a également changé depuis 1998, ils ne cherchent plus à entraver la mise en place du projet même s'ils ne manquent pas de s'interroger sur son positionnement par rapport au FMI (Yuan et Murphy, 2010).

Dix ans après le lancement de l'ABMI, la taille des marchés obligataires en monnaie locale des pays émergents de la région (Japon exclu) a été multipliée par plus de 5,5. Ainsi, en mars 2013, la capitalisation des marchés obligataires en monnaie locale s'élève 6689 milliards de dollars contre 1202 en 2003 (ADB, 2013). Elle est supérieure, en volume, à la capitalisation des marchés libellés en dollars. Les marchés obligataires des pays estasiatiques sont donc en pleine croissance. En mai 2012, lors de la réunion des gouverneurs des Banques Centrales et des Ministres des Finances des pays de l'ASEAN+3, les gouvernements ont décidé de renforcer l'ABMI en mettant en place l'ABMI «New Roadmap + qui affiche trois directions vers lesquelles l'ABMI doit tendre: 1/ produire des résultats tangibles afin de répondre aux problèmes actuels (d'instabilité financière notamment) ; 2 / répondre aux problèmes liés aux marchés obligataires et 3/ s'adapter au marché financier mondial en introduisant la question de la volatilité des flux de capitaux (site ASEAN).

Ce détour par le menu de l'institutionnalisation des relations interétatiques en Asie orientale, permet d'étayer encore l'hypothèse d'un régime régional dit « de collaboration ", comme mode de production d'un BPR. Il permet également de conclure que le processus institutionnel en cours sur le périmètre ASEAN +3 peut être qualifié de «régionalisme en profondeur». L'actualité récente montre de plus que ce processus enregistre une progression incrémentale : depuis la crise de 1997, de nouvelles mesures viennent régulièrement renforcer l'institutionnalisation des liens entre les pays.

L'EPINL a donc ainsi pour valeur ajoutée de permettre une lecture contextualisée de chaque processus régional et d'en expliquer les spécificités historiques. Cette grille théorique présente néanmoins une limite intrinsèque forte en ne proposant pas d'outils quantitatifs pour mesurer notamment l'intensification des flux commerciaux ou financiers dans une zone. Les chercheurs n'ont alors d'autre choix que de mobiliser les outils quantitatifs de l'EI, et d'en interpréter les résultats dans le cadre préalablement établi par l'EPINL. Cette " combinaison ", dont l'éclectisme maîtrisé a déjà été souligné, ne rencontre pas d'impossibilité épistémologique majeure, dans la mesure où les outils quantitatifs convoqués sont considérés comme a-théoriques, ce que va s'attacher à montrer la suite de l'analyse.

\section{Les méthodes quantitatives de l'El en complément de l'EPINL}

Le point précédent a déjà mentionné que la demande d'un régime commercial régional n'était pas aussi pressante en Asie Orientale, que celle d'un régime monétaire. Le régionalisme -construction institutionnelle interétatique- ne concerne donc pas ce domaine. Des travaux ont par ailleurs mis en évidence la faiblesse (voire l'absence) de la régionalisation dans le domaine des flux financiers (Adams, 2008 et Rigg et Schou-Zibell, 2009). Par contre, la régionalisation des flux commerciaux est intense, et l'EPI ne propose pas d'outils permettant d'en rendre compte. 

d'intensité relative ou encore les taux de croissance du commerce intra-régional, permettent, dans un premier temps, de mettre en évidence un processus de régionalisation au sein d'une zone donnée : ils en révèlent l'intensité et le dynamisme. En 2011, les exportations intra-régionales de l'ASEAN+3 et de l'ASEAN+5 s'élèvent respectivement à $36,5 \%$ et à $43 \%$ des exportations totales. La même année, les importations intra-régionales représentent respectivement à $45 \%$ et $51,4 \%$ des importations totales pour les regroupements concernés.

Les taux de croissance du commerce intra-régional au sein de l'ASEAN+3 et de l'ASEAN+5 (cf. Tableaux ci-dessous) montrent par ailleurs le dynamisme des échanges intra-zone, qui augmentent plus rapidement que les échanges avec le reste du monde.

Tableau 1. Comparaison des taux de croissance annuels des exportations régionales de l'ASEAN+3 et de l'ASEAN+5, 1985-2010

\begin{tabular}{|l|l|l|l|}
\hline Taux de croissance & Tx 85-97 & Tx 98-10 & Tx 85-10 \\
\hline Exp intra-A+3 & 13,3 & 10,8 & 12,1 \\
\hline Exp vers RDM & 8,8 & 10,0 & 9,4 \\
\hline Exp intra-A+5 & 12,8 & 9,8 & 11,3 \\
\hline Exp vers RDM & 8,2 & 9,4 & 8,8 \\
\hline
\end{tabular}

Source : calculs des auteures d'après la base de données Chelem

Les flux bilatéraux d'importations et d'exportations, qui ont permis d'effectuer ce calcul, sont exprimés en millions de dollars courants.

Tableau 2. Comparaison des taux de croissance annuels des importations régionales de l'ASEAN+3 et de l'ASEAN+5, 1985-2010

\begin{tabular}{|l|l|l|l|}
\hline Taux de croissance & Tx 85-97 & Tx 98-10 & Tx 85-10 \\
\hline Imp intra-A+3 & 12,7 & 10,9 & 11,8 \\
\hline Imp avec RDM & 9,7 & 10,3 & 10,0 \\
\hline Imp intra-A+5 & 13,0 & 12,2 & 12,6 \\
\hline Imp avec RDM & 9,9 & 9,3 & 9,6 \\
\hline
\end{tabular}

Source: calculs des auteures d'après la base de données Chelem

Dans la littérature, en complément (ou non) d'une analyse statistique "simple ", les modèles de gravité, présentés comme un outil empirique robuste, sont fréquemment employés pour mettre en avant les déterminants du commerce bilatéral et notamment le rôle du biais régional souvent illustré par l'appartenance à une région de référence (Frankel, 1998) ou à un accord régional (Frankel et Wei, 1998 ; Soloaga et Winters, 2001 ; 
Gaulier et al., 2004 ; Fontagné et Zignago, 2007 ; Baier et Bergstrand, 2009). Cette méthode sera utilisée ici pour déterminer le périmètre ayant le plus d'influence sur la direction des échanges intra-régionaux. Cela permettra de compléter économétriquement les résultats obtenus à partir du calcul des taux de croissance et de confirmer ainsi que le regroupement $\mathrm{ASEAN}+3$ constitue bien un périmètre de régionalisation.

\subsection{L'apport méthodologique d'un modèle de gravité à effets spécifiques}

Tinbergen (1962) et Pöyhönen (1963) sont les premiers à appliquer les modèles de gravité au commerce international. Ils donnent une justification intuitive à leur équation gravitationnelle. Ils tentent d'expliquer certaines régularités des flux commerciaux, que la théorie traditionnelle du commerce international ne parvenait pas à expliquer. En faisant référence à la loi de gravité d'Isaac Newton ( $17^{\mathrm{e}}$ siècle), ces auteurs ont formulé l'hypothèse selon laquelle les échanges entre deux pays dépendent de leurs masses économiques respectives (comme facteur d'attraction) et de la distance qui les sépare (comme facteur de résistance). Le volume du commerce entre deux pays doit ainsi augmenter avec la taille du PIB et diminuer avec la distance. Plus qu'une formalisation théorique rigoureuse, leur point de départ était plutôt un raisonnement intuitif ad hoc. À l'instar de ce qui avait déjà été fait dans d'autres domaines, tels que les flux de migration ou le tourisme, ils mobilisent le modèle de gravité afin de mettre en évidence les principaux déterminants du commerce international (Schmiedel, 1998).

Différents auteurs essaieront par la suite d'apporter une justification théorique à l'emploi du modèle de gravité. Leamer (1974), Anderson (1979), Bergstand (1989) et Deardorff (1998) s'appuient sur la théorie dite traditionnelle du commerce international (notamment le modèle Heckscher-Ohlin) pour fournir un cadre théorique au modèle. Helpman et Krugman (1985) puis Helpman (1987) justifient, quant à eux, le modèle de gravité par la nouvelle théorie du commerce international. Au regard de ces différentes études, nous pouvons déduire, à l'instar de Feenstra et al. (2001) que le modèle de gravité peut avoir plusieurs justifications théoriques. Il peut ainsi s'appliquer aussi bien dans le cadre des anciennes théories du commerce international que dans celui des nouvelles, comme dans le cadre de l'EPI. Etant donné son fort pouvoir explicatif, il permet de révéler les principaux éléments de la régionalisation.

\subsection{L'évaluation du biais régional en Asie orientale : mobilisation du modèle de gravité}

Cette méthode est donc ici considérée comme un outil a-théorique, à la disposition des économistes dans le but d'identifier les déterminants de la régionalisation. Afin d'en démontrer la pertinence, elle est ici appliquée au cas asiatique.

Le calcul de l'évolution du taux de croissance du commerce intra-zone ayant permis de mettre en exergue que les regroupements est-asiatiques, ASEAN+3, périmètre d'institutionnalisation des relations après crise et ASEAN+5 (les 13 + Taïwan et Hong Kong), regroupement contenant l'ensemble des pays d'Asie Orientale, constituent bien des zones de régionalisation. La méthode des modèles de gravité vient ensuite compléter ce premier résultat, en évaluant les biais régionaux respectifs de l'ASEAN+3 et de l'ASEAN 
+5 , et, par suite, en révélant l'influence que ces deux périmètres ont sur l'orientation des flux régionaux.

51 Cette étude calcule les coefficients attribués aux deux regroupements est-asiatiques ASEAN+3 et ASEAN+5 sur une période de 21 années (1985-2005). Pour ce faire, elle retient 34 pays (cf. Tableau 3 ) représentant $80 \%$ des exportations et importations mondiales en 2005 et $90 \%$ des exportations et $80 \%$ des importations, de l'ASEAN $+3^{8}$.

Tableau 3. Les 134 pays retenus dans l'équation gravitationnelle

\begin{tabular}{|l|l|}
\hline Région & Pays retenus \\
\hline Asie Orientale & $\begin{array}{l}\text { Les } 10 \text { membres de l'ASEAN, Chine, Corée du Sud, Japon, Hong Kong et } \\
\text { Taiwan }\end{array}$ \\
\hline $\begin{array}{l}\text { Union } \\
\text { Européenne }\end{array}$ & $\begin{array}{l}\text { Allemagne, Autriche, Danemark, Espagne, Finlande, France, Grèce, Irlande, } \\
\text { Italie, Pays Bas, Portugal, Royaume-Uni, Suède }\end{array}$ \\
\hline $\begin{array}{l}\text { Amérique du } \\
\text { Nord }\end{array}$ & États-Unis, Canada, Mexique \\
\hline Asie du Sud & Inde \\
\hline Océanie & Australie, Nouvelle Zélande \\
\hline
\end{tabular}

Ne seront retenus ici que 13 pays pour l'Union Européenne, les derniers élargissements de l'Europe ne seront pas pris en compte. Ces pays ayant peu d'échanges avec l'Asie Orientale, l'impact est négligeable. De plus, la Belgique et le Luxembourg ne figurent pas parmi les pays retenus, les données du FMI pour les flux commerciaux ne différenciant pas les deux pays jusqu'en 1999.

L'équation du modèle de gravité peut dès lors être formulée de la façon suivante :

$\mathrm{X}_{\mathrm{ijt}}=\beta_{1} \mathrm{PIB}_{\mathrm{it}}+\beta_{2} \mathrm{PIB}_{\mathrm{jt}}+\beta_{3} \mathrm{Pibpc}_{\mathrm{it}}+\beta_{4} \mathrm{Pibpc}_{\mathrm{jt}}+\beta_{5}$ Dpibpc $_{\mathrm{ijt}}+\beta_{6}$ Distwces $_{\mathrm{ijt}}+\beta_{7}$ Rem $_{\mathrm{it}}+\beta_{8}$ Rem ${ }_{\mathrm{jt}}+\beta_{9} \mathrm{ADJ}_{\mathrm{ijt}}+\beta_{10}$ LangCom $_{\mathrm{ijt}}+\beta_{11}$ LangEth $_{\mathrm{ijt}}+\beta_{12} \mathrm{~A}+5_{\mathrm{ijt}}+\beta_{13} \mathrm{~A}+3_{\mathrm{ijt}}+\beta_{14} \mathrm{UE}_{\mathrm{ijt}}+\beta_{15} \mathrm{ALENA}_{\mathrm{ijt}}+$ $\mu_{\mathrm{ijt}}$

$\mathrm{M}_{\mathrm{ijt}}=\beta_{1} \mathrm{PIB}_{\mathrm{it}}+\beta_{2} \mathrm{PIB}_{\mathrm{jt}}+\beta_{3} \mathrm{Pibpc}_{\mathrm{it}}+\beta_{4} \mathrm{Pibpc}_{\mathrm{jt}}+\beta_{5} \mathrm{Dpibpc}_{\mathrm{ijt}}+\beta_{6}$ Distwces $_{\mathrm{ijt}}+\beta_{7}$ Rem $_{\mathrm{it}}+\beta_{8}$ $\operatorname{Rem}_{\mathrm{jt}}+\beta_{9} \mathrm{ADJ}_{\mathrm{ijt}}+\beta_{10}$ LangCom $_{\mathrm{ijt}}+\beta_{11}$ LangEth $_{\mathrm{ijt}}+\beta_{12} \mathrm{~A}+5_{\mathrm{ijt}}+\beta_{13} \mathrm{~A}+3_{\mathrm{ijt}}+\beta_{14} \mathrm{UE}_{\mathrm{ijt}}+\beta_{15}$ Où ALENA $_{\mathrm{ijt}}+\mu_{\mathrm{ijt}}$

$\mathrm{X}_{\mathrm{ijt}}$ représente les flux d'exportations du pays i vers le pays $\mathrm{j}$ à la date $\mathrm{t}^{9}$,

$\mathrm{M}_{\mathrm{ijt}}$ les flux d'importations du pays i vers le pays $\mathrm{j}$ à la date $\mathrm{t}$,

PIB $_{\text {it }}$ le PIB du pays $i^{10}$,

$\mathrm{PIB}_{\mathrm{jt}}$ le PIB du pays $\mathrm{j}$,

Pibpc $_{\text {it }}$ le PIB par habitant du pays i,

$\mathrm{Pibpc}_{\mathrm{jt}}$ le PIB par habitant du pays $\mathrm{j}$,

Dpibpc $_{\mathrm{ijt}}$, l'écart de développement économique ${ }^{11}$,

Distwces $_{\mathrm{ijt}}$, la distance entre les deux capitales pondérée par leur poids dans la population totale,

Rem $_{\mathrm{it}}$ l'éloignement du pays i aux marchés alternatifs ${ }^{12}$,

Rem $_{\mathrm{jt}}$ l'éloignement du pays j aux marchés alternatifs,

$\mathrm{ADJ}_{\mathrm{ijt}}$ le fait de partager une frontière terrestre ${ }^{13}$,

LangCom $_{\mathrm{ijt}}$ le fait que les pays i et $\mathrm{j}$ partagent une langue commune,

LangEth $_{\mathrm{ijt}}$ le fait que les pays i et j partagent une langue ethnique, 
$\mathrm{A}+5_{\mathrm{ijt}}$, le fait d'appartenir au regroupement régional ASEAN $+5^{14}$

$\mathrm{A}+3_{\mathrm{ijt}}$, le fait d'appartenir au regroupement régional ASEAN +3

$\mathrm{UE}_{\mathrm{ijt}}$, le fait d'appartenir au regroupement régional UE

ALENA $_{\mathrm{ijt}}$, le fait d'appartenir au regroupement régional ALENA

$\mu_{\mathrm{ijt}}$, une erreur de spécification

Cette équation reprend en grande majorité les variables généralement qualifiées de «naturelles» dans la littérature : la masse économique, la distance économique et la distance géographique. La « distance géographique » mesure la distance entre les deux capitales pondérée par le poids de la capitale dans la population totale de chaque pays (variable nommée ici distwces) ${ }^{15}$. Ce calcul, à la différence d'une variable distance géodésique standard, permet de prendre en compte la répartition géographique de la population et, ainsi, de minorer le poids de la capitale. Ce procédé donne une image plus représentative de la répartition des flux entre les pays. Des variables explicatives qualitatives (frontière commune, langue officielle commune et langue ethnique) sont également intégrées afin d'améliorer le pouvoir explicatif de l'équation. Cette dernière inclut ainsi une variable "distance relative ", qualifiée dans la littérature d'éloignement ou remoteness. L'introduction de cette variable se fonde sur l'hypothèse que les distances relatives entre les pays partenaires influent sur les échanges. En effet, les flux d'échanges bilatéraux peuvent être impactés, non seulement par la distance et la masse économique des deux pays, mais aussi par celles des partenaires commerciaux alternatifs. Deux pays, éloignés géographiquement des autres, commercent généralement plus entre eux que deux pays séparés par la même distance absolue mais géographiquement proches d'autres marchés (Helliwell, 1998; Deardorff, 1998). Comme le soulignent Baldwin et Taglioni (2006), cette variable "remoteness» remplit les mêmes fonctions que la variable « résistance commerciale multilatérale » employée par Anderson et Van Wincoop (2003), permettant ainsi d'éviter une surévaluation des variables régionales et un biais dans l'estimation du terme d'erreur. Pour un pays $\mathrm{i}$ exportant vers un pays $\mathrm{j}$, la variable éloignement des partenaires commerciaux alternatifs, est donc définie par la somme des distances moyennes entre le pays i et les autres pays importateurs, divisée par le PIB des pays importateurs, -c'est-à-dire, pondérée par la masse économique des autres pays importateurs dans l'économie mondiale. Cette variable se calcule en sommant les distances moyennes entre le pays i et les autres pays exportateurs, divisée par le PIB des pays exportateurs. Le même raisonnement est mené pour le pays j importateur.

$$
\begin{aligned}
& \operatorname{Rem}_{\mathrm{it}}=\Sigma_{\mathrm{n}, \mathrm{n \neq j}}\left(D_{\mathrm{ni}} / \mathrm{Y}_{\mathrm{nt}}\right) \\
& \operatorname{Rem}_{\mathrm{jt}}=\Sigma_{\mathrm{n}, \mathrm{n \neq \textrm {i }}}\left(\mathrm{D}_{\mathrm{nj}} / \mathrm{Y}_{\mathrm{nt}}\right)
\end{aligned}
$$

Où $\mathrm{D}$ représente la distance entre les pays, $\mathrm{Y}$ le PIB.

$$
\begin{aligned}
& \text { Cette variable représente ainsi les } \\
& \text { avec des pays autres que le pays } j \text {. }
\end{aligned}
$$

Les trois dernières variables visent enfin à estimer l'importance de la dimension régionale dans les flux commerciaux bilatéraux. Les variables régionales retenues sont l'appartenance aux regroupements ASEAN +3 et ASEAN +5 ainsi que les variables de deux autres accords régionaux emblématiques à l'heure actuelle : l'UE (Union Européenne à 13) et l'ALENA (Accord de libre-échange nord-américain, regroupant les Etats-Unis, le Canada et le Mexique). Ce sont des variables muettes avec un codage binaire, où 1 désigne la présence de relations entre les pays membres des regroupements régionaux ( 1 si deux pays appartiennent au même regroupement) et 0 , l'absence de relations. 
La technique d'estimation par effets spécifiques est retenue pour estimer le modèle (Matyas, 1997 ; Egger et Pfaffermayr, 2003 ; Gaulier et al., 2004 ; Pusterla, 2006, ...). Le test du Multiplicateur de Lagrange (LM) ${ }^{16}$ et le test d'Hausman ${ }^{17}$, figurant dans le Tableau 2, valident l'utilisation de cette spécification. À l'instar d'Ehrmann et al., (2001) et Arthukorola (2008), seuls les effets fixes temporels sont estimés, permettant ainsi de conserver les variables explicatives sans dimension temporelle. L'influence des facteurs invariants dans le temps et propres à chaque pays, sera captée par les variables constantes qui sont introduites.

L'analyse est menée par période, la crise de 1997 pouvant être considérée comme le choc économique ayant le plus fortement affecté les économies est-asiatiques. Le modèle est donc estimé sur une période longue, 1985-2005, et sur deux sous-périodes, 1985-1997 et 1998-2005, ce qui permet d'observer l'évolution des coefficients des variables avant et après crise.

Afin de dépasser le problème de valeurs manquantes ${ }^{18}$, ces dernières sont transformées en valeurs nulles (Eichengreen et Irwin, 1998 ; Tayebi et Hortamani, 2007, ...). L'utilisation du logarithme dans l'équation gravitationnelle ne permettant pas cette transformation, 1 est alors ajouté aux valeurs des flux d'exportations et d'importations. Le logarithme de cet ensemble est ensuite calculé. En d'autres termes, cela équivaut à avoir comme variable à expliquer log $\left(1+\mathrm{X}_{\mathrm{ijt}}\right)$ ou $\log \left(1+\mathrm{M}_{\mathrm{ijt}}\right)$. Cela implique que si $\mathrm{M}_{\mathrm{ijt}}$ ou $\mathrm{X}_{\mathrm{ijt}}=0$, alors log $\left(1+\mathrm{M}_{\mathrm{ijt}}\right)$ ou $\log \left(1+\mathrm{X}_{\mathrm{ijt}}\right)=0$ car $\log (1)=0$.

\subsection{Les résultats du modèle de gravité : la pertinence d'un périmètre économique}

Le modèle de gravité analyse les déterminants de la régionalisation dans un périmètre géographique choisi. Le Tableau 4 synthétise les résultats obtenus avec la méthode des effets spécifiques lors de son application à l'Asie Orientale. Les coefficients du modèle dans ce tableau sont pour la plupart fortement significatifs à moins de $1 \%$. Le $\mathrm{R}^{2}$ qui varie entre 0,82 et 0,84 est relativement fort. Cela signifie que les variations des flux d'exportations ou d'importations sont expliquées à plus de $80 \%$ par les variations des variables explicatives.

- Le poids du PIB du pays exportateur ou importateur pour les deux modalités joue de plus en plus dans l'orientation des flux commerciaux des 34 pays. Ainsi l'accroissement de la taille des pays influe sur les flux commerciaux, résultat compatible avec les résultats attendus des modèles de gravité.

- À l'instar des coefficients des PIB, les coefficients attribués pour les PIB par habitant des pays exportateurs et importateurs pour les deux types de flux sont positifs et significatifs. Le PIB par habitant du pays exportateur dans les deux cas a un impact légèrement plus élevé que celui du pays importateur sur longue période Ainsi l'offre des pays semble jouer une importance plus grande que la demande dans l'intensité des flux commerciaux entre 1985 et 2005.

- La variable mesurant l'écart de développement est significative sur longue période et après 1998. Son coefficient positif est faible, montrant ainsi que les écarts de développement entre les pays ont peu d'impact. Le commerce interindustriel entre les pays semble être faiblement prépondérant. 
Tableau 4. Estimation du biais régional ASEAN+3 et ASEAN+3 pour les flux d'exportations et d'importations

\begin{tabular}{|c|c|c|c|c|c|c|}
\hline \multirow[b]{2}{*}{ Variables } & \multicolumn{3}{|c|}{ Exportations } & \multicolumn{3}{|c|}{ Importations } \\
\hline & 1985.05 & $1985-97$ & $1998-05$ & \begin{tabular}{|l|}
$1985-05$ \\
\end{tabular} & $1985-97$ & 199805 \\
\hline \multirow[t]{2}{*}{ Pibi } & $0.94^{* * * *}$ & $0,87^{* * * *}$ & $0,91^{* * *}$ & $0,89^{* * *}$ & $0,92 * * *$ & $0.99^{* * *}$ \\
\hline & $(172,22)$ & $(123,62)$ & $(110,74)$ & $(164,28)$ & $(227,58)$ & $(118,55)$ \\
\hline \multirow[t]{2}{*}{ Pibj } & $0,88^{* * * *}$ & $0,94^{* * *}$ & $0,99 * * *$ & $0,96^{* * *}$ & $0,87 * * *$ & $0,91 * * *$ \\
\hline & $(661,76)$ & $(333,58)$ & $(119,57)$ & $(277,9)$ & $(221,11)$ & $(110,45)$ \\
\hline \multirow[t]{2}{*}{ Pibpci } & $1,03^{* * * *}$ & $0,94 * * *$ & $0,88 * * *$ & $0.92^{* * *}$ & $1,05 * * *$ & $1,00^{* * *}$ \\
\hline & $(102,7) 8$ & $(75,4)$ & $(54,48)$ & $(92,91)$ & $(81,83)$ & (62,43) \\
\hline \multirow[t]{2}{*}{ Pibpcj } & $0,85 * * *$ & $0.95^{* * * *}$ & $0,89 * * *$ & $0,93^{* * *}$ & $0,87 * * *$ & $0,83 * * *$ \\
\hline & $(84,59)$ & $(75,74)$ & $(55,21)$ & $(93,58)$ & $(67,7)$ & $(51,44)$ \\
\hline \multirow[t]{2}{*}{ Dpibpc } & $0,01^{*}$ & $-0,002$ & $0,04 * * *$ & $0,02 *$ & 0,004 & $0,02^{* *}$ \\
\hline & $(1,83)$ & $(-0,24)$ & $(3,72)$ & $(2,4)$ & $(0,48)$ & $(1,96)$ \\
\hline \multirow[t]{2}{*}{ Districes } & $-0,71 * * *$ & $-0,61^{* * *}$ & $-0,68 * * *$ & $-0,64^{* * *}$ & $-0,68^{* * *}$ & $-0,76^{* * *}$ \\
\hline & $(-35,09)$ & $(-23,71)$ & $(-22,09)$ & $(-32,10)$ & $(-25,72)$ & $(-24,53)$ \\
\hline \multirow[t]{2}{*}{ Remi } & $-0,84 * * * *$ & $-0,58^{* * *}$ & $-0,27 * * *$ & $-0,46 * * *$ & $-0,79 * * * *$ & $-0,92 * * *$ \\
\hline & $(-38,36)$ & $(-20,54)$ & $(-8,12)$ & $(-21,29)$ & $(-27,53)$ & $(-27,75)$ \\
\hline \multirow[t]{2}{*}{ Remj } & $-0,50 * * *$ & $-0,73^{* * * *}$ & $-0,74^{* * *}$ & $-0,73^{* * *}$ & $-0,62^{* * *}$ & $-0,31^{* * *}$ \\
\hline & $(-22,89)$ & $(-26,04)$ & $(-22,20)$ & $(-33,97)$ & $(-21,57)$ & $(-9,29)$ \\
\hline \multirow[t]{2}{*}{ Adj } & $0,55^{* * * *}$ & $0,59 * * *$ & $0,51^{* * *}$ & $0,57^{* * *}$ & $0,55^{* * *}$ & $0,53^{* * *}$ \\
\hline & $(22,69)$ & $(10,54)$ & $(0,82)$ & $(33,3)$ & $(0,66)$ & $(8,17)$ \\
\hline \multirow[t]{2}{*}{ LangCom } & $0,45 * * *$ & $0,38^{* * * *}$ & $0,35 * * *$ & $0,37 * *$ & $0,50 * * *$ & $0,37^{* * *}$ \\
\hline & $(20,09)$ & $(0,6)$ & $(0,17)$ & (8.43) & (8.48) & $(6,49)$ \\
\hline \multirow[t]{2}{*}{ Lange th } & $0,61^{1 * * *}$ & $0,59^{* * * *}$ & $0,50 * * *$ & $0,55^{* * * *}$ & $0,63^{* * * *}$ & $0,59 * * *$ \\
\hline & $(24,59)$ & $(11,09)$ & $(0,78)$ & $(13,39)$ & $(11,49)$ & $(0,24)$ \\
\hline \multicolumn{7}{|l|}{ Biais régional } \\
\hline \multirow[t]{2}{*}{$A+5$} & $0,25^{5+4}$ & $0,1^{9+\ldots+\cdots}$ & $0,38^{\star \star * t}$ & $0,26^{+4+*}$ & $0,211^{+* *}$ & $0,31^{* \star \star *}$ \\
\hline & $(4,94)$ & $(2,96)$ & $(5,00)$ & $(6,32)$ & $(B, 15)$ & $(4,14)$ \\
\hline \multirow[t]{2}{*}{$\begin{array}{c}A+3 \\
\end{array}$} & $0,35^{++4}$ & $0,33^{+* n+}$ & $0,48^{* \star *}$ & $0,39^{+* * t}$ & $0,30^{* * *}$ & $0,42^{\star \star \star *}$ \\
\hline & $(8,08)$ & $(5,99)$ & $(7,38)$ & $(0,27)$ & $(0,25)$ & $(0,47)$ \\
\hline \multirow[t]{2}{*}{$\overline{\mathrm{UT}}$} & $0,46^{* * * *}$ & $0,44^{* * *}$ & $0,31^{* * *}$ & $0,38^{* * * *}$ & $0,52^{* * * *}$ & $0,37^{* * * *}$ \\
\hline & $(0,18)$ & $(0,77)$ & $(3,99)$ & $(0,73)$ & $(0,94)$ & $(4,89)$ \\
\hline \multirow{2}{*}{ ALENA } & $0,29 * *$ & $0,28^{*}$ & $0,59 * * *$ & $0,39^{* * * *}$ & 0,22 & $0,42^{* *}$ \\
\hline & $(2,51)$ & $(1,93)$ & $(3,39)$ & $(3,47)$ & $(1,44)$ & $(2,42)$ \\
\hline$\overline{\text { Adjusted R2 }}$ & 0,83 & 0,82 & 0,84 & 0,83 & 0,82 & 0,84 \\
\hline F-Statistic & $7185,4 * * *$ & $4258,9 * * *$ & $2817,68^{* * * *}$ & $7353,88^{* * * *}$ & $4091,8 * * *$ & $2851,8 * * *$ \\
\hline $\begin{array}{l}\text { Multiplicateur } \\
\text { de La garange }\end{array}$ & 116064,1 & 58244,42 & 22563,76 & 111702,8 & 64336,41 & 22278,38 \\
\hline $\begin{array}{l}\text { Test Hausman } \\
\text { (ddl }=?\end{array}$ & 1288,791 & 339,9284 & 243,535 & 346,0125 & 72,9459 & 209,5103 \\
\hline Observ ations & 23362 & 14586 & 8976 & 23562 & 14584 & 8976 \\
\hline
\end{tabular}

- La variable distance exerce un fort effet négatif sur le volume des échanges d'exportations ou d'importations entre les 34 pays. En d'autres termes, plus les pays sont éloignés entre eux, moins ils commercent. Ce résultat correspond aux résultats classiques de modèles de gravité. À titre d'exemple, pour la période 1985-2005, l'éloignement de 1\% entre le pays i et j entraîne une baisse des exportations de 0,71\%.

- On s'attend généralement à ce que les coefficients des variables éloignement Remi et Remj soient positifs, ce qui n'est pas le cas ici que ce soit pour les pays exportateurs ou importateurs. Ici les variables Remi et Remj jouent dans le sens d'une force répulsive (les coefficients sont tous significatifs au seuil de 1\%). Bien que deux pays soient éloignés géographiquement des autres marchés, cette distance ne favorise pas nécessairement le commerce entre eux. Cela s'explique notamment par la nécessité d'acheter certains biens indispensables pour la consommation primaire de la population ou pour la production nationale.

- La variable adjacence influe positivement sur les échanges conformément aux résultats classiques des modèles de gravité. Il en va de même pour la variable langue commune. Son influence sur les flux d'importations diminue néanmoins après 1998. Il convient de noter que la variable langue ethnique a un coefficient plus important que celui de la variable langue commune. Cela permet de mettre en évidence ici le rôle positif des communautés ethniques dans les échanges commerciaux des 34 pays.

- L'analyse des coefficients des regroupements régionaux permet de montrer une spécificité nordaméricaine dans les échanges internationaux. En effet, les coefficients de la variable ALENA sont significatifs et positifs. L'impact de cet accord dans la direction des flux commerciaux pour les pays membres est plus élevé et significatif depuis 1998. L'accord de libre-échange ayant été signé en 1994, il est normal que ses effets se fassent davantage sentir sur la seconde 
période. Le coefficient de la variable UE est, lui aussi, positif et significatif mais son coefficient diminue sur la seconde période pour les deux flux. Les échanges croissants avec les autres pays européens non encore membres (nouvelle vague d'élargissement aux Pays d'Europe Centrale et Orientale en 2004) font diminuer l'importance de l'impact du regroupement UE à 13 sur les flux commerciaux. Les importations entre deux pays de l'Union Européenne après 1998 sont $48 \%$ plus importantes que «ce qu'elles devraient être », une fois les autres variables contrôlées.

60 Pour les regroupements est-asiatiques l'ASEAN+5 et l'ASEAN+3, deux principaux constats peuvent être faits. Tout d'abord, les coefficients des deux variables sont positifs et significatifs pour les deux flux et sur toutes les périodes. En d'autres termes, cela signifie que les deux regroupements influent positivement sur l'orientation des flux commerciaux. Si les coefficients de ces variables sont comparés, il convient de souligner, dans un second temps, que ceux de l'ASEAN+3 sont légèrement plus élevés pour les deux modalités. Par exemple, le fait d'appartenir à l'ASEAN+3 influe plus sur le sens des exportations que le fait d'appartenir à l'ASEAN+5. Deux pays appartenant au périmètre ASEAN+3 exportent, entre 1985 et $2005,42 \%\left((\exp (0,35)-1)^{*} 100\right)$ de plus que ce qu'ils devraient selon les prévisions du modèle, contre $28,4 \%\left((\exp (0,25)-1)^{*} 100\right)$ pour deux pays appartenant au périmètre ASEAN+5. Il convient de noter que les coefficients des variables ASEAN+3 et ASEAN+5 diminuent sur la période 1985-1997, pour augmenter après 1998, la crise de 1997 semblant avoir impulsé un renforcement des liens commerciaux dans ces périmètres.

61 Ces estimations permettent de montrer que l'ASEAN+3 et l'ASEAN+5 sont des périmètres de régionalisation et que l'influence de l'ASEAN+3 sur l'orientation des flux commerciaux en Asie Orientale apparait comme plus importante que celle exercée par l'ASEAN+5. Elles confirment surtout la pertinence des outils quantitatifs de l'EI pour évaluer un processus de régionalisation.

62 Le périmètre ASEAN+3 étant à la fois le théâtre d'un processus de régionalisation (point 3) et de régionalisme en profondeur (point 2), on peut donc en conclure selon la convention retenue au point 1 qu'il s'agit d'un processus d'intégration régionale en profondeur.

\section{Propos conclusif}

Les arguments développés ont bien permis de progresser dans l'établissement d'une compatibilité effective entre certaines méthodes quantitatives propres à l'économie internationale traditionnelle et des concepts issus de l'une des branches standard de l'EPI : l'EPI institutionnaliste néo-libérale. Ce travail vient ainsi valider l'hypothèse de recherche formulée par Pierre Berthaud dans l'introduction de son HDR (2006). Il y précise en effet : "si l'économie internationale est outillée pour identifier la nature des problèmes d'ordre qui se posent à l'économie mondiale, et sur un plan plus normatif, pour décrire différents types d'issues à ces problèmes, c'est sur le terrain de l'EPI qu'il faut se tourner pour prendre en compte l'hétérogénéité des acteurs (...) et étudier comment se construit leur réponse collective(...).» (p 6). Notre recherche permet ainsi de confirmer l'intérêt de l'EPI pour sa capacité à prendre en compte les déterminants nationaux et les modalités institutionnelles de la coopération internationale, ou régionale. Alors que le modèle gravitaire permet de tester l'hypothèse d'une intensification des flux d'échanges entre des pays géographiquement proches, l'EPI permet d'analyser les motivations d'un éventuel portage institutionnel qui se mettrait en 
place pour accompagner cette intensification. La combinaison des deux approches permet de progresser dans la caractérisation des deux piliers de l'intégration économique régionale définis dans la première partie du texte.

Si l'option retenue ici a été de tester les apports d'une telle approche du fait régional par l'étude du cas asiatique, les résultats obtenus montrent bien que les méthodes quantitatives sont aisément transposables à tout ensemble régional dont le commerce intra-zone est suffisamment développé. Les concepts de l'EPINL permettent également d'appréhender toute forme de coopération institutionnelle interétatique et par conséquent toute coopération régionale. Par suite, l'approche combinée proposée ici permet la comparaison des processus régionaux à la fois dans l'espace et dans le temps comme l'ont suggéré certaines incidentes sur l'intégration européenne.

\section{BIBLIOGRAPHIE}

Adams C., (2008), “Emerging East Asian Banking Systems Ten years after the 1997/98 Crisis”, Working Papers Series on regional economic integration, $\mathrm{n}^{\circ} 16$, Asian Development Bank.

Afmm (Asean+3 Finance Ministers Meeting), (2006), Progress Report of the Asian Bond Markets Initiative (ABMI), November, disponible sur le site Asian Bonds on line, http:// asianbondsonline.adb.org/documents/Progress_Report_ABMI_Nov2006.pdf

Anderson J. (1979), A theoretical foundation of the gravity model, American Economic Review, vol $69, \mathrm{n}^{\circ} 1$.

Anderson J. and Van Wincoop E., (2003), "Gravity with Gravitas: a Solution to the Border Puzzle", American Economic Review, vol. 93, $\mathrm{n}^{\circ} 1$.

Asian Development Bank (ADB), (2009), "Regional Surveillance for Economic Stability”, Asian Economic Monitor, mars.

Asian Development Bank (ADB), (2010a), Institutions for Regional Integration. Toward an Asian Economic Community.

Asian Development Bank (ADB), (2010b), “Exchange Rate Cooperation: Is East Asia Ready?”, Asian Economic Monitor, décembre.

Asian Development Bank (ADB), (2013), Asian Economic Monitor, June.

Athukorala P.C., (2008), “China's integration into global production networks and its implications for export-led growth strategy in other countries in the region", Working Paper, $n^{\circ}$ 2008/04, The Australian National University.

Aubin C., (2002), « Biens publics et régionalisation », The European Union Review, vol.10 N¹, pp 57-74.

Baier S L. et Bergstrand J.H. (2009), "Estimating the effects of free trade agreements on international trade flows using matching econometrics", Journal of International Economics, vol. 77, $n^{\circ} 1$, p. 63-76, February. 
Baldwin R.E. et Taglioni D., (2006), “Gravity for dummies and dummies for gravity equations”, Discussion Paper, ${ }^{\circ} 5850$, Centre for Economic Policy Research.

Beeson M., (2003), “ASEAN plus three and the rise of Reactionary Regionalism”, Contemporary South East Asia, vol.25, n², august.

Benaroya F., (2002), « Organisations régionales et gouvernance mondiale », dans Jacquet P. et al. (Dir.) Gouvernance mondiale, rapport du CAE, pp. 431-448.

Bergstrand J., (1989), “The Generalized Gravity Equation, Monopolistic Competition, and the Factor-Proportions Theory in International Trade", The Review of Economics and Statistics, Vol. 71, $\mathrm{n}^{\circ} 1$, pp. 143-153.

Berthaud P., (2006), Intégration économique et gouvernance internationales : un programme de recherche en économie politique internationale (EPI), HDR, Université Pierre Mendès-France Grenoble, LEPII, 54 p.

Bhagwati J. (2002), Free Trade Today, Princeton University Press.

Boidin B. \& Zuindeau B., (2006), « Socio-économie de l'environnement et développement durable : état des lieux et perspectives », Mondes en développement, $\mathrm{n}^{\circ}$ 135, pp .7-37.

Boisseau du Rocher S., (1998), ASEAN et la construction régionale en Asie du Sud-est, Edition L'Harmattan, Paris.

Boulanger E., (2006), « Le Japon et l'Appel de l'Asie », Cahiers de Recherche du CEIM, n06-05, mars.

Bresling S., (2008), "Towards a Sino-centric regional order? Empowering China and constructing regional order(s)", in Dent C. (Ed.) China, Japan and Regional Leadership in East Asia, Edward Elgar Editions.

Chavagneux C., (2010), L'économie politique internationale, col. Repère, La Découverte, 126 p.

Chey H-K., (2009), "The Changing Political Dynamics of East Asian Financial Cooperation: The Chiang Mai Initiative”, Asian Survey, vol. 49, n³, mai-juin, pp. 450-467.

Cook L. \& Sachs J., (2002), « Les biens collectifs régionaux dans le cadre de l'assistance internationale ", dans Kaul I., Grunderger I. et Stern M. (dir) Les biens publics à l'échelle mondiale. La coopération internationale au XXIème siècle, Oxford University Press.

Crockett A., (1997), “Why is Financial Stability a Goal of Public Policy?” Economic Review, Fourth Quarter.

Deardorff A.V., (1998), “Determinants of Bilateral Trade: Does Gravity Work in a neoclassical world?”, in Frankel J.A. (dir.) The Regionalization of the World Economy, NBER, University of Chicago Press.

Egger P. \& Pfaffermayr M., (2003), “The Proper panel econometric specification of the gravity equation: A three-way model with bilateral interaction effects", Empirical Economics, vol 28.

Ehrmann M., Gambacorta L., Martinez-Pagès J., Sevestre P. and Worms A., (2001), "Financial Systems and the Role of Banks in Monetary Policy Transmission in the Euro Area", European Central Bank Working Paper Series, $\mathrm{n}^{\circ} 105$, December.

Eichengreen B. et Irwin D.A., (1998), "The Role of History in Bilateral Trade Flows", in Frankel J.A. (Ed.) The Regionalization of the World Economy, NBER, University Chicago Press.

Feenstra R.C., Markusen J.R., Rose A.K., (2001), “Using the gravity equation to differentiate among alternative theories of trade", Revue Canadienne d'Economie, vol 34, n², mai. 
Figuière C. et Guilhot L. (2008), « La Chine, prochain leader économique de l'Asie Orientale ? ", Région et Développement, $\mathrm{n}^{\circ} 28$.

Figuière C. et Guilhot L., (2007), « Vers une typologie des processus régionaux : le cas de l'Asie Orientale », Revue Tiers Monde, n¹92, octobre-novembre, pp. 897-917.

Figuière C. et Guilhot L., (2011), « La Chine : avenir du découplage asiatique ? », dans Diemer A. et Dozolme S. (dir.). Les enseignements de la crise des Subprimes, Editions C. Juglar.

Fontagné L. \& Zignago S., (2007), “A re-evaluation of the impact of regional agreements on trade patterns”, Economie Internationale, $n^{\circ} 109$, août.

Frankel J.A. \& Wei S-J., (1998), Regionalization of World Trade and Currencies, in Frankel J.A. (dir) The Regionalization of the World Economy, NBER, University Chicago Press.

Frankel J.A. (1998), The Regionalization of the World Economy, NBER, University Chicago Press.

Gabas J.-J. et Hugon P. (2001), Les biens publics mondiaux et la coopération internationale, L'Économie politique, no 12, p. 19-31.

Gaulier G, Jean S. and Unal-Kensenci D., (2004), Regionalism and the Regionalisation of International Trade, Working Papers, CEPII, novembre.

Gilpin R., (2001), Global Political Economy. Understanding the international economic order, Princeton University Press, $416 \mathrm{p}$.

Guilhot L. (2012) Le régionalisme commercial en Asie Orientale. Une évaluation par un modèle de gravité à données de panel, Revue économique, vol 63, n6, novembre, pp 1179-1192

Guilhot L. (2008), L'intégration économique régionale de l'ASEAN+3. La crise de 1997 à l'origine d'un régime régional, Thèse, UPMF, Grenoble, disponible sur http://tel.archives-ouvertes.fr/ tel-00348467/en/.

Guilhot L. (2009), «L'impact de la crise de 1997 sur l'ASEAN+3 : les apports d'une approche en EPI », Mondes en Développement, n¹47, 3ème trimestre.

Guilhot L. (2010), Assessing the impact of the main East-Asian free trade agreements using a gravity model. First results, Economics Bulletin, vol. 30, Issue 1.

Hamanaka S. (2009), Re-considering Asian Financial Regionalism in the 1990s, ADB Working paper series on regional economic integration, $n^{\circ} 26,18 \mathrm{p}$.

Helliwell J.F. (1998), How much do National Borders Matter?, The Brookings Institution.

Helpman E. \& Krugman P. (1985), Market Structure and Foreign Trade, MIT Press, Massachusetts.

Helpman E. (1987), Imperfect Competition and International Trade: Evidence from Fourteen Industrial Countries, Journal of the Japanese and International Economies, $\mathrm{n}^{\circ} 1$.

Henning C. R., (2009), The future of the Chiang Mai Initiative: An Asian Monetary Fund?, Policy Brief, Peterson Institute for International Economics, number PB09-5, February.

Higgot R., (1998), The Asian Economic Crisis: A Study in the Politics of Resentment, New Political Economy, vol.3; Iss.3.

Hugon P., (2001), « Analyse comparative des processus d'intégration économique régionale », Etude de la Direction Générale de la Coopération Internationale et du Développement, Ministère des Affaires Etrangères.

Jetin B., (2009), « L'intégration monétaire asiatique : dollarisation, monnaie commune ou simple coopération monétaire? », Revue Tiers Monde, n 199, pp. 591-610. 
Kawai M., (2004), "Regional Financial Stability as a Regional Public Good", Tokyo Forum on the Operational Dimensions of Supplying Regional Public Goods through Regional Development Assistance, October.

Kébabdjian G. (1998), « La théorie de la régulation face à la problématique des régimes internationaux ", L'année de la régulation, vol.2, p. 101-127.

Kébabdjian G., (1994), L'Economie mondiale, Enjeux nouveaux, nouvelles théories, Editions Seuil. Kébabdjian G., (2006), « De l'économie internationale à l'économie politique internationale », introduction de Berthaud et Kébabdjian (Dir.) La question politique en économie internationale, Collections Recherche, la Découverte.

Kehoane R.O. (1984), “After Hegemony: Cooperation and Discord in the World Political Economy”, Princeton UP.

Kindleberger C.P., (1986), International Public Goods without International Government, American Economic Review, vol 76, $\mathrm{n}^{\circ} 1$.

Krasner S.D., (1983), International Regimes, Ithaca, Cornell, UP.

Krugman P.R. (1991) Geography and Trade, MIT.

Leamer E., (1974), The Commodity Composition of International Trade in Manufactures: An Empirical Analysis, Oxford Economic Papers, vol. 26, n³, November, pp. 350-374.

Matyas L., (1997), "Econometric Specification of the Gravity Model”, The World Economy, vol. 20, n 3.

Nicolas F., (2006), « Intégration économique en Asie de l'Est : les progrès limités de l'approche institutionnelle », dans Boisseau du Rocher S. et Godement F. (dir.) Asie entre pragmatisme et attentisme, Les Etudes de la Documentation française, édition 2006-2007.

Ocampo R., (2002), “The Role of Regional Institutions”, in Debek O., Flassbeck H., Heymann D., Jenkins P. Lipschitz L., Ocampo J.A, Park Y.C, Vincze J. White W. et Wyplosz C. (Eds.) A regional approach to financial crisis prevention-Lessons from Europe and initiatives in Asia, Latin America and Africa, FONDAD Publication.

Plummer M. G. \& Wignaraja G., (2006), "The post-crisis sequencing of economic integration in Asia: Trade as a complement to a monetary future", Economie internationale, $n^{\circ} 107$, pp. 59-85.

Plummer M. G., (2010), “Regional Monitoring of Capital Flows and Coordination of Financial Regulation: Stakes and Options for Asia", ADBI Working Papers Series, $\mathrm{n}^{\circ} 201$.

Pöyhonen P., (1963), "A tentative Model for the Volume of Trade between Countries", Weltwirtschaftliches Archiv, vol 90.

Pusterla F., (2006), "Regional Integration Agreements: Impact, Geography and Efficiency”, Third CEPII-IDB Conference, The New Regionalism: Progress, Setbacks and Challenges, Washington, Février.

Rigg R. \& Schou-Zibell L., (2009), “The Financial Crisis and Money Markets in Emerging Asia”, Working Papers Series on regional economic integration, $n^{\circ} 38$, Asian Development Bank, November.

Schmiedel F., (1998), L'orientation géographique des échanges commerciaux de la Chine, Thèse de Sciences Economiques, Université d'Auvergne.

Siregar R. et Chabchitrchaidol A., (2013), "Enhancing the Effectiveness of CMIM and AMRO: Selected Immediate Challenges and Tasks", ADBI Working Paper, $\mathrm{n}^{\circ} 403$, January.

Soloaga I., Winters L.A, (2001), “Regionalism in the nineties: what effect on trade?", The North American Journal of Economics and Finance, vol. 12, $\mathrm{n}^{\circ} 1$. 
Stein A., (1983), “Coordination and Collaboration. Regimes in an Anarchic World”, in Krasner S. (Ed.) International Regimes, Ithaca: Cornell University.

Stiglitz J., (1999), “Knowledge as a Global Public Good”, in Kaul I. et al. (Eds.) Global Public Goods: International Cooperation in the 21st Century, United Nations Development Program, Oxford University Press, pp.b308-325.

Sussangkarn C., (2010), “The Chiang Mai Initiative Multilateralization: Origin, Development and Outlook", ADBI Working Papers Series, $\mathrm{n}^{\circ} 230$.

Tayebi S.K. \& Hortamani A., (2007), “The Impact of Trade Integration on FDI Flows : Evidence from the EU and ASEAN+3", in Kim J-K and Ruffini P-B. (Eds.) Corporate Strategies in the Age of Regional Integration, Edward Elgar.

Tinbergen J., (1962), Shaping the world economy. Suggestions for an international policy, Twentieth Century Fund, New York.

Winkler A., (2010), “The Financial Crisis: a Wake-up Call for Strengthening Regional Monitoring of Financial Markets and Regional Coordination of Financial Sector Policies?", ADBI Working Papers Series, $\mathrm{n}^{\circ} 199,52 \mathrm{p}$.

Wyplosz C., (2002), « L'instabilité financière internationale », in Kaul I., Grunderger I. et Stern M. (dir.) Les biens publics à l'échelle mondiale. La coopération internationale au XXIème siècle, Oxford University Press, (1999) pour la version en langue anglaise.

Yuan W.J \& Murphy M., (2010), "Regional monetary Cooperation in East Asia. Should the United States be Concerned?", Center for Strategic \& International Studies, November.

\section{NOTES}

1. Une première version de ce texte a fait l'objet d'une communication au colloque Économie politique internationale et nouvelles régulations de la mondialisation, qui s'est tenu à Poitiers en mai 2009. Les auteures remercient les rapporteurs de la revue pour leur lecture approfondie qui a permis à ce texte de progresser.

2. Cette analyse porte sur les 13 pays membres de l'ASEAN+3. Les membres fondateurs de l'ASEAN (Association des Nations du Sud-est asiatique, créée en 1967), sont l'Indonésie, la Malaisie, les Philippines, Singapour, la Thaïlande. Ils ont été rejoints par le Sultanat de Brunei (1984), le Vietnam (1995), le Laos et la Birmanie (1997), le Cambodge (1999). Le Japon, la Chine et la Corée $\mathrm{du}$ Sud sont les «trois" pays qui accompagnent l'Association depuis 1998. Nous faisons également référence dans la partie 3 de ce texte au regroupement ASEAN+5 qui rassemble les 13 de l'ASEAN+3 ainsi que Taiwan et Hong Kong.

3. L'ALENA, accord de libre-échange nord-américain, a été signé en 1994, entre les États-Unis, le Canada et le Mexique.

4. Pour une analyse détaillée des accords de libre-échange en Asie, notamment l'impact de l'AFTA et de l'accord ASEAN-Chine sur le commerce intra-régional, voir Guilhot (2012).

5. Dans la mesure où aucun consensus n'émerge à ce jour sur la reconnaissance du leadership de la Chine pas plus que du Japon, ce texte ne développera pas les arguments qui amènent à ce résultat. Sur ce thème se référer à Figuière et Guilhot (2008).

6. "Ten years ago, when Mr. Sakakibara was Vice Minister, he proposed the creation of an Asian Monetary Fund (AMF) in response to the 1997/98 financial crisis. The proposal was rejected, Mr. Sakakibara said, because of a strong opposition from the United States, as well as a lack of consultation by the Japanese government with its Asian neighbors - especially People's Republic of China (PRC)". (Brève ADB, 15 mai 2009). 
7. Si à l'origine, seuls $20 \%$ des « droits de tirage » d'un pays donné pouvaient être débloqués sans l'accord du FMI, depuis mai 2012, le seuil est monté à 30\% et devrait atteindre 40\% en 2014 (site de l'ASEAN, 21 octobre 2013).

8. Calculs des auteures d'après les données du FMI, Statistical Yearbook, divers numéros.

9. Données recueillies dans FMI, Direction Trade of Statistics Yearbook.

10. Données recueillies dans World Economic Outlook Database, site FMI à l'adresse suivante : http://www.imf.org/external/pubs/ft/weo/2006/02/data/index.aspx

11. Calcul des auteures (Différence absolue entre le PIB par habitant des pays i et j).

12. Calcul des auteures $\left(\operatorname{Rem}_{\mathrm{ijt}}=\Sigma_{\mathrm{n}, \mathrm{n} \neq \mathrm{j}}\left(\mathrm{D}_{\mathrm{ni}} / \mathrm{Y}_{\mathrm{nt}}\right)\right)$.

13. Les variables (distance, adjacence, langue officielle et langue ethnique) proviennent de la base de données du CEPII (Centre d'Études Prospectives et d'Informations Internationales), http://www.cepii.fr/francgraph/bdd/distances.htm

14. Calcul des auteurs (codage binaire, appartenance au regroupement, 1, sinon, 0 ).

15. La dénomination du CEPII est ici reprise, cette variable étant disponible sur la base de données de ce dernier.

16. Test confirmant la présence d'effets spécifiques

17. Test déterminant l'indépendance des effets spécifiques vis-à-vis des variables explicatives

18. L'utilisation des données compilées du FMI ne permet pas de dire si ces données ne sont pas disponibles, trop faibles pour être répertoriées, ou si les pays ne commercent pas ensemble.

\section{RÉSUMÉS}

Les processus régionaux se caractérisent par une double réalité : économique et politique. Ces deux dimensions se combinent de manière très différente en fonction du lieu et du moment. Ce papier a pour objectif de valider l'hypothèse de l'opérationnalité de la combinaison des concepts issus de l'Économie Politique Internationale Néo-Libérale (EPINL), et des outils quantitatifs de l'Économie Internationale (EI), pour rendre compte de cette diversité. Très éloigné du cheminement européen, le processus asiatique constitue un test intéressant. En effet, les membres de l'ASEAN + 3 n'ont à ce jour signé aucun accord de libre-échange multilatéral régional alors même que cette zone enregistre une forte intensification des flux intra-régionaux. Ces pays développent par contre certaines formes de coopérations interétatiques, dans le domaine monétaire et financier notamment, en dépit de l'absence de régionalisation dans ce domaine. La combinaison méthodologique envisagée va s'avérer épistémologiquement soutenable, tout en permettant une lecture de toute forme de processus régional.

Regional processes are characterized by a dual reality: economic and political. These two dimensions combine in very different ways depending on the place and the time. This paper aims to validate a methodology based on a combination of concepts from the Neoliberal International Political Economy and quantitative tools of International Economics, to account for this diversity. Very different from the European process, the Asian one is an interesting test for this methodology. Indeed, the ASEAN + 3 members don't have signed a regional multilateral free trade agreement even though the intra-regional flows are very high in this area. Nevertheless, these countries develop an interstate cooperation in the monetary and financial sector, despite the lack of regionalization in this sector. The proposed methodological combination will be epistemologically sustainable, while allowing a reading of all forms of regional process. 
INDEX

Mots-clés : intégration régionale, Asie orientale, économie politique internationale, économie internationale, bien public régional, modèle de gravité

Keywords : regional integration, East Asia, international political economy, international economics, regional public good, gravity model

Code JEL C23 - Panel Data Models • Spatio-temporal Models, F13 - Trade Policy • International Trade Organizations, F59 - Other, 053 - Asia including Middle East

\section{AUTEURS}

\section{CATHERINE FIGUIÈRE}

Université Grenoble Alpes, Centre de recherche en économie de Grenoble (CREG)

\section{LAËTITIA GUILHOT}

Institut d'administration des entreprises (IAE) Lyon et Université Grenoble Alpes, Centre de recherche en économie de Grenoble (CREG) 